\title{
Phytate solubilizing microorganisms and enzyme phytase to combat nutritional problems in cereal- based foods
}

\begin{abstract}
Phytic acid (myo-inositol hexakisphosphate) is the main storage form of phosphorus (P) in plants and accounts for more than $80 \%$ of the total $\mathrm{P}$ in cereals. It is one of the sources of energy and an important mineral chelating compound in plants. However, it is a major anti-nutritional factor for human beings especially to individuals consuming large quantities of cereals like rice, finger millet and wheat. The anti-nutritional properties of phytates are known to be reduced through dephosphorylating process by means of enzyme, 'phytase' of microbial origin. Numerous studies have demonstrated the effectiveness of microbial phytase in improving the utilization and absorption of nutrients from phytate. Reduced level of phytate in foods is known to enhance the bioavailability of minerals and other associated nutrients needed for human health. Phytase is getting prime importance in processing and manufacturing industries that supply feeds for birds and non-ruminant animals. It can be a good substitute for inorganic phosphorus (Pi) supplements in poultry and animal feed, which otherwise can cause environmental pollution. Research has been conducted to prove anti-nutritional nature of phytic acid along with an effort to reduce its content in foods consumed. This review briefly narrates the study related to properties of phytate, application phytate solubilizing microorganisms and enzyme 'phytase' obtained from them.
\end{abstract}

Keywords: phytic acid, phytase, phytate solubilizing microorganisms, nutrition, pollution, phosphorus, anti-nutritional, birds, feeds, non-ruminant, animal feed, solubilizing, inorganic phosphorus, germination, triglycerides
Volume 4 Issue 3 - 2017

\author{
Savita PD,' Yallappa M,' Nivetha $N,{ }^{2}$ Suvarna \\ VCl \\ 'Department of Microbiology, University of Agricultural \\ Sciences, India \\ ${ }^{2}$ Department of Agricultural Microbiology, University of \\ Agricultural Sciences, India
}

Correspondence: Savita PD, Department of Agricultural Microbiology, University of Agricultural Sciences, India - 560012 , Tel 9108023603915 , 91 7795900025,

Email savitapd02@yahoo.com

Received: February 02, 2017 | Published: March 16, 2017

\section{Introduction}

One of the most important constituents of crops is phytic acid (myoinositol hexakisphosphate). Phytate is a complex organic compound abundantly found within the plant system. It is known to accumulate in higher quantity during maturation of plants and their seeds. It acts as a competitor, especially for adenosine triphosphate (ATP) production during the rapid biosynthesis of phytin at seed maturity and during inhibition of seed metabolism, inducing dormancy. ${ }^{1}$ Phytate serves as phosphorus $(\mathrm{P})$ reserve and as an energy store, mainly during seed germination. It regulates the readily available seed/plant inorganic phosphorus (Pi) levels during further growth. Phytate is considered as a strong metal (cations) chelating compound as its inositol sixphosphate (IP6) carries twelve negative charges. ${ }^{2}$ Mechanism of phytic acid binding to other monovalent, divalent or trivalent cations leading to insoluble complexes has been démonstrated by Reddy et al. ${ }^{3}$ According to researchers, phytic acid has certain important functions in the animal and human body as well. It may inhibit the production of hydroxyl radicals; act as an antioxidant to normalize cell or represent as a cancer fighter; reduce blood clots, cholesterol and triglycerides that circulate throughout the blood stream. ${ }^{4}$ Though believed to be a useful component, its content in excess would be undesirable for our consumption. Phytate are not digestible by non-ruminant animals as well as human beings as their digestive system lacks the enzyme to solubilize them. Hence, the undigested phytate in the small intestine negatively affect absorption of minerals and other nutrients. ${ }^{5}$ It inhibits utilization of certain digestive enzymes in the intestinal tract of animals and human beings. Negative charges of the phytic acid results in binding of other organic compounds like carbohydrates and vital proteins required for energy and growth. ${ }^{6}$ Thus, its property as an antinutritional factor overshadows its benefits. Sufficient trials have not been conducted to prove the role of phytic acid in human beings or animals. This review highlights on the disadvantages of high phytic acid in human or animal diet and centres on the significance of phytate solubilizing microorganisms or its phytase.

\section{Discussion}

\section{Anti-nutritional properties of phytic acid (phytate)}

Phytate exists widely in food grains belonging to the class of cereals, oil seeds and nuts. It accounts to about $3 \%$ to $5 \%$ of the dry weight of plant seeds. ${ }^{7}$ Cultivation of cereals represents the highest agricultural areas across the world. Most of the cereals are low in proteins and minerals but high in starch and phytate. Phytic acid could be one of the attributing reasons to malnutrition in Asian countries where upto $75 \%$ of the total calorie intake is from cereals. ${ }^{8}$ This cause can contribute to illnesses like muscle cramping, weak blood circulation, fatigue or anaemia in women and children. Phytate are known to chelate calcium out of the human body by reducing its access to minerals required for bone building process and nerve transmitting areas. ${ }^{9}$ Zinc and iron are essential for the immune system. Phytic acid derivatives have shown high affinity towards iron [III]. ${ }^{10}$ The most widely recognised strategy by people to improve micronutrient absorption is supplementation with pharmaceutical vitamins and minerals in the form of capsules, syrups and peels. Supplementation with synthesized nutrients may eventually lead to new health problems. Therefore, there is a need for natural substitute to overcome micronutrient deficiencies in our body. 


\section{Properties of phytase and its application}

Phytases (myo-inositol hexakisphosphate phosphohydrolases) belong to special class of enzymes called acid phosphatases. It can hydrolyze highly stable phytic acid to free myo-inositol phosphates releasing chelated components as well as five phosphorus units from the complex. ${ }^{11}$ The enzyme shares a highly conserved sequence motif found at the active sites of acid phosphatases. Phytase is widespread in nature and is known to be occurring in every plant system and in extensive groups of microorganisms. Phytase has been discovered in bacteria, yeasts and moulds that are in environment as well as in the animal gut. In higher plants, phytase occur predominantly in grains, seeds and pollens. ${ }^{12}$ It gets activated in plant based foods during processing like soaking, seasoning, cooking, fermentation and acidification. Acidic $\mathrm{pH}$ plays an important role in enzyme maturation and activation. The $\mathrm{pH}$ requirement for induction of phytase ranges from 4.2 to $5.5 .{ }^{13}$ Phytase has been reported to be responsible for phytate solubilization during germination too to make phosphate, minerals and myo-inositols available for plant growth and development. ${ }^{3}$ It improves the nutritional value of plant foods by enhancing protein digestibility and mineral availability through phytate hydrolysis during digestion in the intestinal tract. ${ }^{9}$ Phytase have been cloned and characterized, such as fungal phytase from Aspergillus ficuum, bacterial phytase from Escherichia coli ${ }^{9}$ and Bacillus subtilis. ${ }^{3}$

Hydrolysis of phytic acid to less phosphorylated myo- inositol derivatives is important in human and animal diet. It improves nutritional value of plant based foods by enhancing protein digestibility and mineral availability through phytate hydrolysis during food processing as well as during digestion and absorption in the intestinal region. ${ }^{9}$ Research has proved that microbial phytases are most promising for biotechnological applications. ${ }^{14}$ Since phytase is proven to hydrolyze phytic acids and their salts effectively, purification and characterization of novel phytase can be of significance in food and feed processing industries. Previously studied phytase enzyme of Aspergillus sp. has already received commercial value in the poultry feed market. But industries that supply pelleted phytase enzyme are under scrutiny to check the effectiveness, stability and shelf life of enzyme at different environmental conditions. Enzymes are known to be sensitive not only to high temperatures but also to chemicals and $\mathrm{pH}$ fluctuations. Thermostability of this enzyme is an important factor during the preparation of feed pellets. It should be stable to resist inactivation by heat during feed processing and storage. Feed pelleting is generally done at temperature as high as $95^{\circ} \mathrm{C}$. Although, phytase inclusion using an after spray apparatus for pellet diets and /or chemical coating of phytase may help to overcome the heat destruction of the enzyme, thermostable phytase will still be a suitable candidate for feed supplements. ${ }^{15}$ According to Wyss et al., ${ }^{16}$ the effectiveness and limitations of phytase supplementation to animal feed depends on substrate specificity. In vitro feeding trials have revealed that phytase with broad substrate specificity are better suited for animal nutrition purposes than with narrow substrate specificity. Broad substrate specificity of an enzyme can readily solubilize phytate from any sources to myo-inositol monophosphates. When used as feed additives, it should be effective in releasing phytate phosphorus and associated nutrients/minerals in the digestive tract, only then commercial production of enzyme can prove economical to feed industries. It should be stable during feed processing as well as storage and must be able to resist inactivation by heat. Thus, considering these properties, commercial production of phytase can prove economical to feed industries. But it all involves cost factor However an alternative and cost-effective solution would be direct use of non- pathogenic or probiotic phytate solubilizing degrading microorganisms. The raw feed should be fermented with efficient phytase producing yeasts or bacteria and then subjected to high temperatures feed processing and storage. However it is believed that phytase activity obtained directly from microorganisms is tolerant and sometimes performs even better at high temperatures. Research has proved that phytase directly from microorganisms can be most promising for biotechnological applications. There are several reports regarding improved ionizable ions due to phytase. Experiments conducted by Vandenberg et al., ${ }^{17}$ proved significant enhancement of mineral mobilization; approximately $20-28 \%, 26-37 \%$ and $24-42 \%$ of $\mathrm{Zn}^{2+}, \mathrm{Fe}^{2+}$ and $\mathrm{Ca}^{2+}$, respectively after the addition of phytase enzyme to chickpea flour. There was significant reduction (75-88\%) in phytic acid content. Purified phytases obtained from Aspergillus niger used in wheat bran flour have reported to increase Iron absorption in human beings. ${ }^{18}$ However, phytases obtained from Aspergillus ficuum are already in the market for years and have proved to be of significant value in animal feed supplements.

\section{Abundance of phytate solubilizing microorganisms and its application}

Phytate degrading/solubilizing microorganisms have been isolated from the rhizosphere, phyllosphere and spermosphere. Some species are inhabitants of various foods and food products. Phytase producing microorganisms have been isolated from various ecosystems. Microbial phytase activity in soil has been more frequently detected in fungi followed by bacteria. There is no sufficient data to conclude that actinomycetes are one of the efficient phytate degrading organisms. Most of the soil bacteria detected to possess phytase activity belonged to the group g- proteobacteria. Researchers have screened over 2000 microorganisms isolated from soil for phytase production. Most of the positive isolates produced intracellular phytases. However extracellular phytase activity has been observed mostly in fungi. Shieh and Ware ${ }^{19}$ used culture enrichment technique to isolate phytase-producing microorganisms from soil. They found number of Aspergillus niger group that produced extracellular phytases dephosphorylating calcium phytate in acidic solution. A soil isolate, A. ficuum produced the most active phytase in a corn starch-based medium. Phytase from Aspergillus sp. has received commercial value and is being sold in the market to supplement poultry feeds. Sreedevi and Reddy ${ }^{20}$ isolated phytate solubilizing microorganisms from rhizosphere, cowshed and poultry soils. Several isolates showed positive for phytase production and Bacillus sp. was discovered as one of the efficient phytase producer. El-Toukhy et al., ${ }^{21}$ isolated phytase producing organisms from soil and one was identified morphologically and confirmed by 16SrRNA as Bacillus subtilis- MJA. It revealed high phytase activity. Singh et al.,22 isolated thirty two phytase producing bacteria from different soils on phytase screening medium (PSM). Some were identified as Bacillus subtilis by 16 SrDNA. Many researchers have isolated and identified $B$. subtilis as an effective phytatse producer. It is proved to be non-pathogenic and safe for commercial production of phytase. This organism has several other beneficial properties like antibiosis and organic acid producer that can solubilize phosphates in soil.

Phytate is not hydrolysable quantitatively in the human or animal gut unless degraded by gut microbiota. Bifidobacteria is a gut microbiota that selectively colonizes the intestinal tract of 
breast-fed infants and is also relevant colonic bacteria in adults. ${ }^{23}$ This group is considered to be one of the major microbial stimuli for newborns and one of the most important probiotic culture.. In vitro experiments found Bifidobacteria as a good phytase producer. Enriching infant foods with fructo-oligoseccharides (FOS) as prebiotic and with selected bacterium strain as probiotics would be a good supplement in phytate rich food. ${ }^{24}$ Phytase produced in the digestive tract can be very efficient in degrading phytate and improve availability of plant- $\mathrm{P}$ sounds better than vegetables phosphorus to ruminants as demonstrated by Rodehutscord. ${ }^{25}$ The phytase enzyme produced by bacteria and yeasts are extracellular as well as intracellular, however extracellular are more appropriate. ${ }^{26}$ The ability to degrade phytate by five different strains of Bifidobacterial species (Bifidobacterium animalis, B. bifidum, B. infantis, B. longum and B. pseudocatenulatum) isolated from the intestinal tract was evaluated by Haros et al. ${ }^{24}$ These organisms exhibited good solubilizing capacity under in vivo conditions. However Bifidobacteria needs to be cultured and experimented under anaerobic condition which becomes one drawback to work in normal laboratory conditions. Hirimuthugoda et al., ${ }^{27}$ isolated several yeast strains from intestinal tract of sea cucumbers and found some yeasts with good phytase activity. Two yeasts strains that produced high amounts of extracellular and cell bound phytases were identified as, Yarrowia ipolytica and Candida tropicalis. These yeasts could be used as probiotic yeasts by the sea cucumber farming industry. ${ }^{28}$ They studied phytase activity of phytate degrading bacteria that were previously isolated from the gastrointestinal tract of Atlantic cod, Gadus morhua to determine its effect on head kidney leukocytes. Out of the 216 bacterial strains tested, the two phytase producers were identified as Pseudomonas sp. and Psychrobacter sp. based on 16SrDNA analysis. Using certain yeasts species as probiotic microorganisms would probably help to improve food quality. Phytase has been detected in various bacteria and yeasts isolates not only from soil or intestinal tract but from various food sources too. Naturally fermenting foods can be one of the sources to isolate non-pathogenic phytate degrading microorganisms. Ouattara et al., ${ }^{29}$ isolated several phytase producing strains of lactic acid bacteria from pearl millet fermented gruel, 'ben-saalga'. They found two isolates Lactobacillus plantarum and Lactobacillus fermentum with high phytase activity. Several yeast strains were isolated from cereal based food and beer. ${ }^{30}$ Strains from cereal foods identified with good phytase activity were Saccharomyces cerevisiae, S pastorianus, $S$ bayanus, Kazachstania exigua (earlier named Saccharomyces exiguus), Candida krusei ( a teleomorph Issachenkia orientalis) and Arxula adeninivorans. The beer-related strains, S. pastorianus and $S$. cerevisiae were also reported as phytase-positive and were recorded with high level of extracellular phytases, suggesting them to be the strains for the production of wholemeal bread with high content of bio available minerals. Sumengen et al., ${ }^{31}$ isolated Lactobacillus brevis from fermented foods. Several fungal isolates from grains and other plant sources have been isolated and screened as phytase producers. Due to their easy cultivation and high production of extracellular enzymes, filamentous fungi are one of best sources of phytase for use in the feed industry Haefner et al. ${ }^{32}$

All commercial phytase preparations contain microbial enzymes produced by fermentation. Recent research in this field focuses on technical improvement of food processing and better mineral absorption. Direct utilization of phytase producing microbes through fermentation of food products have been tried by many investigators.
Two stage tempeh preparations were tried by $\mathrm{Chen}^{33}$ to simultaneously solubilize phytic acid and soy protein with high efficiency. Fermentation of soy product with co-inoculation of Aspergillus oryzae and A. ficuum resulted in higher phytic acid solubilization than A. oryzae fermentation alone. Askelson et al., ${ }^{2}$ investigated phytate solubilization by fermentation with a novel probiotic mechanism using recombinant Lactobacillus cultures that expressed phytase phyA of Bacillus subtilis. Expression of B. subtilis phytase (in vitro) increased phytate solubilization ability of $L$. acidophilus, $L$. gasseri and $L$. gallinarum approximately by 4,10 and 18 folds over the background activity of empty vector transform ants respectively. Phytase expressing L. gallinarum and L. gasseri were administered to broiler chicks (in vivo) that were fed with a phosphorus deficient diet. Phytase expressed in L. gasseri improved weight gain of broiler chickens to a level comparable to the chickens fed with controlled and adequate diet of phosphorus, thus leading to a conclusion that administration of phytate solubilizing probiotic cultures can improve the performance of livestock animals. ${ }^{2}$

\section{Conclusion}

Since phytate phosphorus in the plant based feeds is not readily available in case of animals and poultry breeding, there is increasing amounts of inorganic phosphorus (Pi) supplementation to meet the requirement. The unutilized feed phosphorus (supplemented with Pi) is being largely excreted by the animals and poultry birds into the environment. This can cause adverse ecological consequences in the long term like eutrophication. Use of phytase or non-pathogenic phytate degrading microorganisms might perhaps replace artificial supplementation. Considering the human diet, particularly expecting and lactating women or growing infants, there is an ample need of bio available calcium, iron and zinc. Fermentation of raw foods with probiotic phytate solubilizing microorganisms (like lactic acid bacteria or yeasts) followed by cooking can prove valuable, avoiding supplementation by synthetic pharmaceutical or nutraceutical products. Although, phytate is reported to be an antioxidant with anticancer activity, its negative effect related to nutrient deficiency is much more severe, especially in economically backward and developing countries. ${ }^{34}$ Fermentation has several other benefits; it can reduce digestive problems; reduce food allergies and improve immune system. Food fermentation practice will possibly prevent deficiencies or malnutrition problems in the long term.

\section{Acknowledgements}

None.

\section{Conflict of interest}

The author declares no conflict of interest.

\section{References}

1. Cosgrove DJ, Irving CJ. Inositol Phosphates:Their chemistry Biochemistry and Physiology. USA:Elsevier; 1980. p. 191-200.

2. Askelson TE, Campasino A, Lee JT, et al. Evaluation of PhytateDegrading Lactobacillus Culture Administration to Broiler Chickens. Appl Environ Microbiol. 2014;80(3):943-950.

3. Reddy NR, Pierson MD, Sathe SK, et al. Interactions of Phytate with Proteins and Minerals: Phytases in legumes and cereals. USA: CRC press; 1989. p. 57-70. 
4. Tran TT. Thermostable Phytase from a Bacillus sp, Heterologous Production, Mutation, Characterization and Assay Development. Sweden: Lund University; 2010. p. 1-126.

5. Bohn T, Davidsson L, Walczyk T, et al. Phytic acid added to white wheat bread inhibits fractional apparent magnesium absorption in humans. Am J Clin Nutr. 2004;79(3):418-423.

6. Coulibaly A, Kouakou B, Chen J. Phytic Acid in Cereal Grains: Healthy or Harmful Ways to Reduce Phytic Acid in Cereal Grains and Their Effects on Nutritional Quality. Am J Plant Nutr Fert. 2011;1:1-22.

7. Lei XG, Porres JM. Phytase Enzymology, Applications and Biotechnology. Biotechnol Lett. 2003;25(21):1787-1789.

8. Sands DC, Morris CE, Dratz EA, et al. Elevating optimal human nutrition to a central goal of plant breeding and production of plant-based foods. Plant Sci. 2009;117(5):377-389.

9. Greiner R, Konietzny U. Phytase for Food Application. Food Technol Biotechnol. 2006;44:125-140.

10. Iemma F, Cirillo G, Gianfranco S, et al. Removal of Metal Ions from Aqueous Solution by Chelating Polymeric Microspheres Bearing Phytic Acid Derivatives. European polymer J. 2008;44:1183-1190.

11. Kornegay ET, Qian H. Replacement of Inorganic Phosphorus By Microbial Phytase for Young Pigs Fed on a Maize Soybean Meal Diet. Bri J Nutr. 1996;76(4):563-578.

12. Greiner R, Konietzny U, Jany KD. Phytate -An Undesirable Constituent of Plant Based Foods. J für Ernährungs medizin. 2006;8:18-28.

13. Singh NK, Joshi DK, Gupta R. Isolation of Phytase Producing Bacteria and Optimization of Phytase Production Parameters. Jundishapur $J$ Microbio. 2014;6:1-6.

14. Pandey A, Szakacs G, Soccol CR, et al. Production, Purification and Properties of Microbial Phytases. Bioresource Technol. 2001;77(3):203214.

15. Konietzny U, Greiner R. Bacterial phytase: Potential application, in vivo function and regulation of its synthesis. Braz J Microbiol. 2004;35:11-18.

16. Wyss M, Brugger R, Kronenberger A, et al. Biochemical Characterization of Fungal Phytases (MyoInositol Hexakisphosphate Phosphohydrolase):Catalytic Properties. Appl Environ Microbiol. 1999;65(2):367-373.

17. Vandenberg CJ, Kumar R, Wilson DM, et al. Orthophosphate Therapy Decreases Urinary Calcium Excretion and Serum 1, 25-Dihydroxy Vitamin D Concentrations in Idiopathic Hyper Calciuria. J Clin Endocrinol Metab. 1980;51(5):998-1001.

18. Sandberg AS, Hulthen LR, Turk M. Dietary Aspergillus niger Phytase Increases Iron Absorption in Humans. J Nutr. 1996;126(2):476-480.

19. Shieh TR, Ware JH. Survey of Microorganisms for the Production of Extracellular Phytase. Appl Microbiol. 1968;16(9):1348-1351.
20. Sreedevi S, Reddy BN. Isolation Screening and Optimization of Phytase Production from Newly Isolated Bacillus spC 43. Int J Pharm Biol Sci. 2012;2:218-231.

21. El Toukhy NM, Youssef AS, Mikhail GM. Isolation, Purification and Characterization of Phytase from Bacillus subtilis MJA. African $J$ Biotechnol. 2013;12:2957-2967.

22. Singh NK, Joshi DK, Gupta RK. Isolation of Phytase Producing Bacteria and Optimization of Phytase Production Parameters. Jundishapur J Microbiol. 2014;6:1-6.

23. Harmsen HJ, Wildeboer Veloo AC, Raangs GC, et al. Analysis of Intestinal Flora Development in Breast Fed and Formula Fed Infants by Using Molecular Identification and Detection Methods. J Pediatr Gastroenterol Nutr. 2000;30(1):61-67.

24. Haros M, Bielecka M, Sanz Y. Phytase Activity as a Novel Metabolic Feature in Bifidobacterium. FEMS microbiol Lett. 2006;247(2):321-239.

25. Rodehutscord M. Current Phosphorus Evaluation Systems for Livestock in Germany. Lohmann-Inf. 2001;25:17-24

26. Konietzny U, Greiner R. Bacterial phytase: Potential application, in vivo function and regulation of its synthesis. Braz J Microbiol. 2004;35:11-18.

27. Hirimuthugoda NY, Chi Z, Wu L. Probiotic Yeasts with Phytase Activity Identified from the Gastrointestinal Tract of Sea Cucumbers. SPC Beche de Mer Information Bulletin. 2007;26:31-33.

28. Lazado CC, Marlowe C, Caipang A, et al. Responses of Atlantic Cod Gadusmorhua Head Kidney Leukocytes to Phytase Produced by Gastrointestinal Derived Bacteria. Fish Physiol Biochem. 2010;36(4):883889.

29. Ouattara SL, Mouquet Rivier C, Icard-Verniere C. Enzyme Activities of Lactic Acid Bacteria from a Pearl Millet Fermented Gruel (Ben-Saalga) of Functional Interest in Nutrition. Int J Food Microbiol. 2008;128(2):395400

30. Nuobariene L, Hansen AS, Jespersen L, et al. Phytase Active Yeasts from Grain Based Food and Beer. J Appl Microbiol. 2011;110(6):1370-1380.

31. Sumengen M, Dincer S, Kaya A. Phytase Production from Lactobacillus brevis. Turk J Biol. 2012;36(5):533-541.

32. Haefner S, Knietsch A, Scholten E, et al. Biotechnological Production and Applications of Phytase. Appl Microbiol Biotechnol. 2005;68(5):588-597.

33. Chen L. Detoxification and Nutritional Enhancement of Soy Meal via Microbial Bioprocessing. USA: Kansas State University; 2013.

34. Ford JA, Colhoun EM, Mcintosh WB, et al. Biochemical Response of Late Rickets and Osteomalacia to a Chupaty Free Diet. Brit Med J. 1972:3:446-447. 